\title{
Evidence of Plasmodium falciparum infection after 12 years from the exposure: cryptic malaria or recrudescence?
}

Ildebrando Patamia ( i.patamia@unict.it)

University of Catania: Universita degli Studi di Catania https://orcid.org/0000-0001-5228-7830

Elisa Cappello

Umberto I di Siracusa Hospital: Ospedale Umberto I di Siracusa

Maddalena Calvo

University of Catania: Universita degli Studi di Catania

Giuseppe Migliorisi

University of Catania: Universita degli Studi di Catania

Antonina Franco

Umberto I di Siracusa Hospital: Ospedale Umberto I di Siracusa

\section{Case report}

Keywords: malaria, recrudescence, Plasmodium falciparum, cryptic malaria

Posted Date: September 17th, 2021

DOI: https://doi.org/10.21203/rs.3.rs-903065/v1

License: (9) This work is licensed under a Creative Commons Attribution 4.0 International License.

Read Full License 

after 12 years from the exposure: cryptic malaria or recrudescence?

Elisa Cappello ${ }^{1}$, Maddalena Calvo ${ }^{2}$, Giuseppe Migliorisi' ${ }^{2}$, Antonina Franco ${ }^{1}$ and Ildebrando Patamia ${ }^{2,3}$

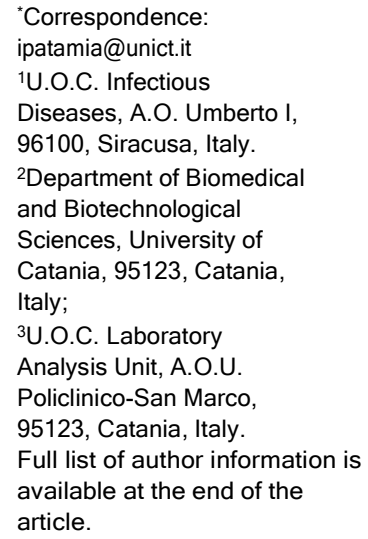

\begin{abstract}
Background: Malaria infections affect a high percentage of the world's population, especially in tropical and subtropical regions. Specifically, Plasmodium falciparum is the most relevant species involved in the etiopathogenesis of this infection. The duration of $P$. falciparum infections is often undefined, as some reported episodes of suspected recrudescence occur several years after initial exposure.
\end{abstract}

Case presentation: We present a case report of malaria infection with low parasitaemia in a man whose last stay in an endemic region or contacts with the local population was twelve years ago. The patient recovered fully with adequate antimalarial treatment, but some aspects of his clinical history were not clearly defined.

Conclusions: We discuss here the possibility that this is either a $P$. falciparum recrudescence or an episode of cryptic malaria, as we cannot carefully verify some details of our patient's life and history.

Keywords: malaria, recrudescence, Plasmodium falciparum, cryptic malaria.

\section{Background}

Malaria is a major public health problem affecting a high percentage of the world's population, especially in tropical and subtropical regions. The modern world has effective antimalarial drugs and insecticides, but people there are still at risk although the high infection rates affect mostly low-income countries, especially Africa [1]. There are several species involved in human infection with similar life cycles, but they may differ in their ability to persist in the human body in the form of relapse or recrudescence. Relapses are cases whose symptoms appear months to years after the primary infection has cleared, due to exoerythrocytic stages (hypnozoites). Recrudescence, on the other hand, can be defined as acute illness due to residual parasitaemia after at least 1-12 months after initial exposure to the etiologic agent, most commonly in Plasmodium falciparum malaria [2]. $P$. falciparum is most dangerous due to severe clinical complications and high morbidity and mortality rates [3]. According to the literature, the duration of $P$. falciparum infections cannot be accurately determined and has been controversial since the first health policy on eradication was applied (the 1950s). Although these infections generally do not last longer than one year, some case reports document suspected recrudescence episodes after one to thirteen years following the elimination period of the primary infection. There are many hypothesized reasons leading to $P$. falciparum persistence, such as incomplete anti-malarial treatment or the presence of a less virulent antigenic variant of the parasite. [4,5] 
Here, we report a case of a possible $P$. falciparum recrudescence in a young man, twelve years after his last exposure to the plasmodial parasite. The patient's clinical symptoms were not suggestive of malaria infection, and the anamnestic data were not consistent with a recent stay in an endemic area for malaria. Aware of the possibility of recrudescence or relapse, we requested a blood sample, which confirmed the presence of $P$. falciparum infection. After complete anti-malarial treatment, the patient was fully recovered.

\section{Case presentation}

On day 1, a 32-year-old African man comes to the emergency room of the Umberto I Hospital in Siracusa with fever, chills and general malaise. The patient was born in Chad but moved to Italy in 2009. He states that he has been in Italy since 2009 and has never returned to his home country in the last twelve years. The patient does not indicate any allergies or previous illnesses, except for a past malaria episode (2005) that resolved spontaneously without treatment. He also refers no contact with people from malaria endemic areas or stays inside or next to airport stations. A chest computed tomography scan (CT) documents pulmonary microembolism and an abdomen CT scan shows hepatomegaly and mesenteric lymphadenopathy. Laboratory data show moderate monocytosis $\left(1500 / \mathrm{mm}^{3}\right.$ with a leukocyte count of $11100 / \mathrm{mm}^{3}$ ), a C-reactive protein (PCR) value of $111 \mathrm{mg} / \mathrm{L}$, and a D-dimer level of $400 \mathrm{ng} / \mathrm{mL}$. In addition, liver and renal function tests show normal values. Surgical consultation precludes necessary intervention. Evidence of pulmonary microembolism leads the patient to the cardio-thoracic department of the hospital. Broad-spectrum antibiotic therapy with ceftriaxone (2 $\mathrm{g} /$ day), anticoagulant treatment with fondaparinux, and antipyretic treatment with acetaminophen (as needed) are prescribed. On day 3 , fever $\left(38.5^{\circ} \mathrm{C}\right)$ and PCR levels $(120 \mathrm{mg} / \mathrm{L})$ remain high, requiring infectious disease consultation. The patient appears alert and oriented, with a sore abdomen and normal blood pressure. Integration of antibiotic treatment with levofloxacin ( $2 \mathrm{~g}$ iv/day) is suggested. Collection of surveillance samples (nasal and throat swabs, stool and urine samples, peripheral venous blood) appears essential. A serum sample is required to perform all of the following serologic tests for a possible infection: IgM and IgG profiles of cytomegalovirus (CMV), Epstein-Barrvirus (EBV), SARS-CoV-2, Toxoplasma gondii, and Rickettsia spp; Vidal-Wright serologic diagnosis; serologic diagnosis of syphilis; serologic profiles of hepatitis $B$ virus (HBV) and hepatis $C$ virus (HCV). Informed consent from the patient for Human Immunodeficiency Virus (HIV) serologic testing is required. Possible previous contact with Mycobacterium tuberculosis will be investigated by Manteaux reaction and QuantiFERON testing. A stool sample will be collected to perform fecal calprotectin dosing, and daily laboratory testing will be integrated with procalcitonin, erythrocyte sedimentation rate (ESR), and protein electrophoresis. A panel for autoimmunity syndromes will also be performed. Finally, due to the patient's home country and another opinion of the parasitologist of University Hospital from Catania, it also seems necessary to investigate the possibility of malaria relapse caused by hypnozoites of the species Plasmodium 
vivax or Plasmodium ovale. A blood sample is sent to Catania for a rapid antigen test and microscopic diagnosis with thick drop and thin smear. Bacterial sepsis is suspected due to a high level of procalcitonin $(5 \mathrm{ng} / \mathrm{mL})$ and persistence of fever $\left(38.5^{\circ} \mathrm{C}\right)$. Therefore, antibiotic treatment is modified: ceftriaxone and levofloxacin are replaced by meropenem ( $3 \mathrm{~g} /$ day) and linezolid (1200 mg/day). A few hours later, the rapid antigen test for malaria shows positive results for either pan-malarial aldolase antigen or Plasmodium falciparum histidine-rich protein 2. After adequate time for microscopic observation, the thick droplet confirms the presence of a plasmodial parasite with a low parasitaemia rate $(4000$ parasites $/ \mathrm{mL})$ and the thin smear shows annular trophozoites (Figure 1), normal sized erythrocytes, Maurer's dots and rare banana-shaped gametocytes (Figure 2). The final diagnosis is $P$. falciparum malaria. This result is confirmed by two further tests, performed 8 and 12 hours after the first test, respectively. The new evidence highlights the need for antimalarial treatment with piperaquine dihydroartemisinin (300-40 $\mathrm{mg} /$ day). Antibiotic treatment with meropenem and linezolid will continue. On day 4, all surveillance cultures show negative results and no tuberculous reactivity is documented. All serologic infection tests and fecal calprotectin dosing are also negative. Inflammatory indices return to normal values. A repeat blood check for malaria parasitaemia is performed 24 hours after the initial detection of infection. Microscopic examination reveals only a moderate presence of $P$. falciparum banana-shaped gametocytes. On day 5 , a new blood sample is taken 24 hours from the last one. This test shows a completely negative result for malaria infection. The autoimmunity syndrome panel shows high rates $(1 / 160)$ for either antinuclear antibodies (ANA) or extractable nuclear antigens (ENA). According to this new data, rheumatology consultation is required and mixed connective tissue disease (MCTD) is diagnosed. On day 6 , the patient is transferred to the General Internal Medicine department of the hospital due to improvement in his cardio-thoracic condition. New surveillance cultures are required to monitor the patient's overall condition. On day 10, a blood culture shows a positive result for Enterococcus faecium, although the patient is afebrile, asymptomatic, and with normalized inflammatory indices. Therefore, antibiotic treatment is modified: meropenem is removed from therapy, and linezolid becomes the sole antibiotic treatment. A control blood culture is requested on day 17, which shows a negative result five days later. On day 25, the patient appears to be fully recovered and is discharged from the hospital based on better laboratory tests and normal chest and abdominal CT scans. On day 39, according to current World Health Organization (WHO) guidelines, an appointment is made for the patient at the hospital's infection center 2 weeks after discharge to check his general condition, blood count, and liver and kidney functions. Chest and abdominal CT scans show no clinical abnormalities, indicating complete resolution of the previous pulmonary microembolism. In addition to these improvements, laboratory tests document complete recovery of the patient. Blood samples obtained on days 3-4-5 were sent to Italian Major Health Institute in Rome for diagnostic confirmation. After this further diagnostic confirmation, the patient was included in an official database of Italian cryptic malaria cases. 


\section{Discussion}

167

168

169

170

171

172

173

174

175

176

177

178

179

180

181

182

183

184

185

186

187

188

189

190

191

192

193

194

195

196

197

198

199

200

201

202

203

204

205

206

207

208

209

210
Plasmodium species that infect humans show a similar multistage life cycle characterized mainly by two stages: an initial liver stage and subsequent proliferation in the blood [1]. Species differences, however, arise either from the virulence rate or from the potential persistence after the acute phase in the human host. Thus, malaria relapses usually occur when a dormant exoerythrocytic stage (hypnozoite) produced by Plasmodium ovale, and Plasmodium vivax regains its infectivity after a variable dormancy period (1-12 months) in the liver. As is well known, hypnozoite production is the most common cause of malaria relapses, but it is not the only condition that leads to malaria recurrence after primary infection. For example, Plasmodium malariae is incapable of producing a liver dormant stage but can remain with a low parasitaemia for a long time (maximum 50 years) even in the absence of symptomatic infection, especially after inadequate antimalarial treatment [6]. In addition, Plasmodium falciparum has been documented in the literature to cause episodes of suspected recrudescence at least a decade after the last exposure, even though this species is not capable of producing hypnozoites [7]. It is difficult to recognize risk factors for possible $P$. falciparum recrudescence. $A$ hypothesis suggests factors like pregnancy, comorbidities (such as diabetes), head injury, HIV infection, anaemia, physical stress and prior incomplete antimalarial treatment [4]. Among these conditions, the main reason leading to $P$. falciparum persistence seems to be incomplete anti-malarial treatment, which prevents eradication of the parasitic infection and also has modulating effects on parasitaemia [4]. According to our patient's history, no treatment was taken for the past malarial episode, so this causal hypothesis was discarded together with diabetes, HIV infection, anaemia and head injury. Consequently, only possible physical stress could be related to our patient. Some other scientific data have speculated about the correlation between $P$. falciparum persistence and the presence of some antigenic variants of the parasite. By attempting an immune escape strategy, $P$. falciparum can produce antigenic variants that are different from the original infectious strain. The antigenic variants differ from each other by variable expression of vargenes encoding PfEMP1 proteins. Although some variants are capable to evade the immune response, they are less able to bind receptors and form erythrocytic adhesions. However, they are mostly expressed in tissues, resulting in a less severe clinical impact of erythrocytic sequestration. Therefore, less virulent antigenic variants may persist in the post-elimination period with minimal or absent clinical manifestations. It is reasonable to assume that some variants develop a decreased fitness to allow a long persistence in the human host [5]. This hypothesis could be important background for further studies: it would be ideal to look for possible less virulent $P$. falciparum variants in every doubtful case of malaria. Some suspected $P$. falciparum recrudescence cases can be classified by the definition of cryptic malaria, which, according to the Centers for Disease Control and Prevention (CDC), can be defined as "a case of malaria in which epidemiological investigations cannot identify an obvious mode of acquisition (this term applies mainly to cases found in non-endemic countries)" [8]. Consequently, cryptic 
malaria can be said to occur when any of the following conditions are present: acute infection with unreliable patient travel history, diagnostic delays, suspected importation of an infected mosquito, or transmission through contact with infected blood or tissue [9]. However, risky conditions as transfusion, transplant, intravenous drug intake, stays near airports, travels and contacts with people from endemic areas were denied by the patient. Our study aims to shed more light on a doubtful case, whose origin is not entirely certain, and to investigate whether it is cryptic malaria or a recrudescence of $P$. falciparum. Following this assumption, there are some interesting points about the epidemiological and clinical statement of our patient. He left Chad, an endemic region for malaria, twelve years ago. His recovery was caused by atypical symptoms not directly associated with malaria infection. His condition was characterized by high levels of procalcitonin and low parasitaemia due to $P$. falciparum. It is well established that procalcitonin is often high in uncomplicated malaria, especially when there is a long interval between the first symptoms and the diagnosis [10]: this aspect supports the suspicion of malaria infection in our patient, who had a high procalcitonin value in the absence of any signs of sepsis. It seems possible to suspect a transient immunodepression in our patient, probably due to a stressful life situation (during a business trip) and to an autoimmune syndrome. Immunodepression may be related to either suspected. $P$. falciparum recrudescence or transient translocation of $E$. faecium from the gastrointestinal tract into the bloodstream. A positive blood culture occurring several days after malaria diagnosis never resulted in an overt sepsis state and no further positive tests followed. Therefore, the high procalcitonin level was only related to the malaria episode. The patient appeared to be reliable denying all the possible predisposing conditions for cryptic malaria. We have not had the opportunity to fully verify every detail of his life and medical history, but we have chosen to believe that his statements are not inaccurate or false. Accordingly, we are confident that this may be one of the rare cases of $P$. falciparum recrudescence, 12 years after the last exposure, with low parasitaemia. Nevertheless, there is a small percentage of uncertainty about the patient's travel history, which leads us to consider the possibility of cryptic malaria. According to all of these observations, it seems to be useful to integrate currently Infectious Diseases Society of America (IDSA) guidelines for the management of fever of unknown origin (FUO) [11] a blood sample for malaria diagnosis within the established therapeutic algorithm (physical examination, imaging tests, laboratory studies such as surveillance cultures and blood cultures). This suggestion arises from the minimal invasiveness of blood collection and the low cost of malaria diagnostic methods. Through this policy, it could be possible to solve some patients' clinical conditions with a derisory expense and a little stressful condition for the patients. For this reason, we want to suggest the execution of malaria screening for all patients with FUO, regardless of geographic origin, considering that the malaria transmission ways are not always clearly recognizable. 


\section{Appendix}

\section{$274 \quad$ Abbreviations}

ESR: erythrocyte sedimentation rate; PCR: C-reactive protein; CT: computerized tomography; CMV: Cytomegalovirus; EBV: Epsteinvirus; SARS-CoV-2: Severe Acute Respiratory Syndrome-Coronavirus-2; HBV: hepatitis B Virus; HCV: hepatis C Virus; HIV: human Immunodeficiency Virus (HIV); ANA: anti-nuclear antibodies; ENA: extractable nuclear antigens; MCTD: mixed connective tissue disease; WHO: World Health Organization; CDC: Centers for Disease Control and Prevention; FUO (Fever of unknown origin); IDSA Infectious Diseases Society of America.

\section{Acknowledgements}

We would like to thank the Italian Major Health Institute in Rome, with particular regards to doctor Daniela Boccolini for her help and competence.

\section{Authors' contributions}

Study concept and design IP, MC, GM; care of the patient EC, IP; bibliography research: IP, MC, GM; drafting the manuscript IP, MC

GM; critical comments on the manuscripts IP, EC; final approval of the version submitted IP. All authors approved the final manuscript.

Funding

This study did not receive any funding.

Availability of data and materials

Data and materials used during the study are available from the corresponding author on reasonable request.

Ethics approval and consent to participate

A written informed consent was required from the patient for the publication of this case report anonymously.

\section{Consent for publication}

Not applicable.

\section{Competing interests}

The authors declare none competing of interests.

\section{Author details}

1 U.O.C. Infectious Diseases, A.O. Umberto I, 96100, Siracusa, Italy;

2 Department of Biomedical and Biotechnological Sciences, University of Catania, 95123, Catania, Italy;

3 U.O.C. Laboratory Analysis Unit, A.O.U. Policlinico-San Marco, 95123, Catania, Italy.

\section{References}

1. Sato S. Plasmodium-a brief introduction to the parasites causing human malaria and their basic biology. J Physiol Anthropol. 2021;7;40(1):1

2. Buck E, Finnigan NA. Malaria. StatPearls. Treasure Island, 2021

3. Zekar L, Sharman T. Plasmodium falciparum malaria. StatPearls. Treasure Island, 2021.

4. Ashley EA, White NJ. The duration of Plasmodium falciparum infections. Malar J, 2014;13:500.

5. Jensen AR, Adams Y, Hviid L. Cerebral Plasmodium falciparum malaria: The role of PfEMP1 in its pathogenesis and immunity, and PfEMP1-based vaccines to prevent it. Immunol Rev, 2020; 293(1):230-252.

6. Grande R, Antinori S, Meroni L, Menengon M, Severini C. A case of Plasmodium malariae recurrence: recrudescence or reinfection? Malar J, 2019; 14;18(1):169.

7. Monteiro W, Brito-Sousa JD, Elizalde-Torrent A, Botto-Menezes C, Melo GC, Fernandez-Becerra C, Lacerda M, Del Portillo HA. Cryptic Plasmodium chronic infections: was Maurizio Ascoli right? Malar J, 2020;30;19(1):440.

8. https://www.cdc.gov/malaria/glossary.html. Accessed 27 June 2021.

9. Cryptic malaria guidance - Travel and Migrant Health Section, HPS: Colindale, 2011.

10.Uzzan B, Izri A, Durand R, Deniau M, Bouchard O, Perret GY. Serum procalcitonin in uncomplicated falciparum malaria: a preliminary study. Travel Med Infect Dis, 2006; 11.4(2):77-80.

11. Wright WF, Auwaerter PG. Fever and Fever of Unknown Origin: Review, Recent Advances, and Lingering Dogma. Open Forum Infect Dis, 2020; 2;7(5). 
Figures

Figure1. A P. falciparum ring (a) and extraterrestrial (b) trophozoites forms.

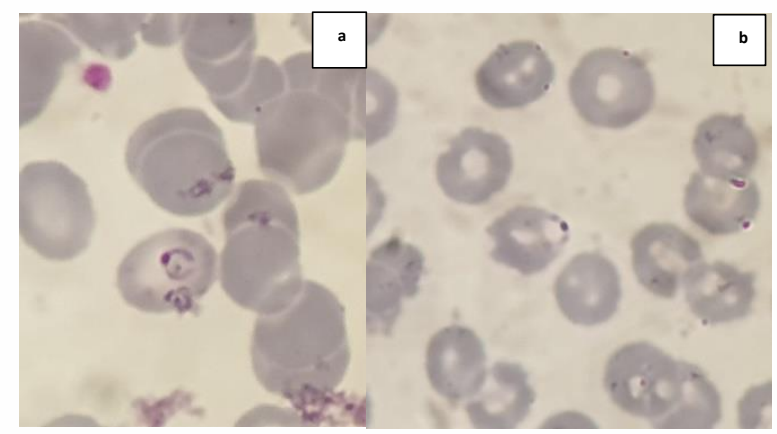

368

Figure2. A P. falciparum banana-shaped gametocyte.

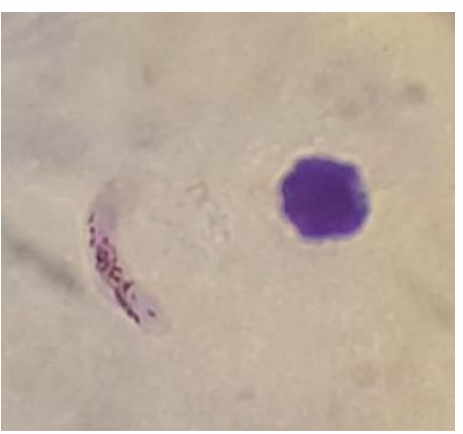


